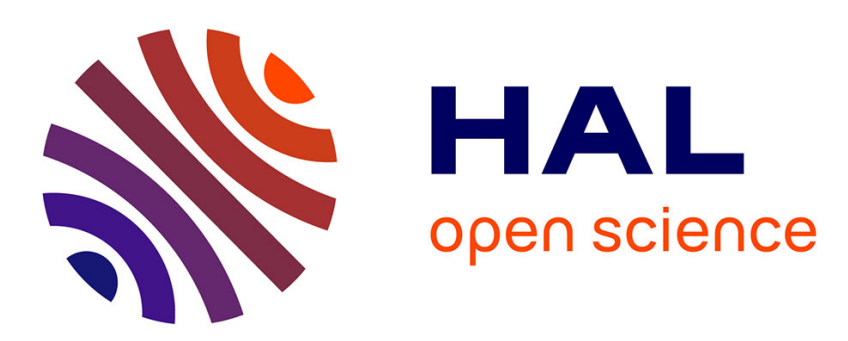

\title{
Fiducial points extraction and charactericwaves detection in ECG signal using a model-based bayesian framework
}

\author{
Mahsa Akhbari, Mohammad B. Shamsollahi, Christian Jutten
}

\section{To cite this version:}

Mahsa Akhbari, Mohammad B. Shamsollahi, Christian Jutten. Fiducial points extraction and charactericwaves detection in ECG signal using a model-based bayesian framework. ICASSP 2013 - 38th IEEE International Conference on Acoustics, Speech and Signal Processing, May 2013, Vancouver, Canada. pp.1257-1261. hal-00839419

\section{HAL Id: hal-00839419 https://hal.science/hal-00839419}

Submitted on 28 Jun 2013

HAL is a multi-disciplinary open access archive for the deposit and dissemination of scientific research documents, whether they are published or not. The documents may come from teaching and research institutions in France or abroad, or from public or private research centers.
L'archive ouverte pluridisciplinaire HAL, est destinée au dépôt et à la diffusion de documents scientifiques de niveau recherche, publiés ou non, émanant des établissements d'enseignement et de recherche français ou étrangers, des laboratoires publics ou privés. 


\title{
FIDUCIAL POINTS EXTRACTION AND CHARACTERISTIC WAVES DETECTION IN ECG SIGNAL USING A MODEL-BASED BAYESIAN FRAMEWORK
}

\author{
Mahsa Akhbari ${ }^{1,2} \quad$ Mohammad B. Shamsollahi ${ }^{1} \quad$ Christian Jutten $^{2}$ \\ ${ }^{1}$ BiSIPL, Sharif university of Technology, Department of Electrical Engineering, Tehran, Iran \\ ${ }^{2}$ GIPSA-Lab, Grenoble, and Institut Universitaire de France, France \\ mahsa.akhbari@gipsa-lab.grenoble-inp.fr, mbshams@sharif.edu, Christian.Jutten@inpg.fr
}

\begin{abstract}
The automatic detection of Electrocardiogram (ECG) waves is important to cardiac disease diagnosis. A good performance of an automatic ECG analyzing system depends heavily upon the accurate and reliable detection of QRS complex, as well as $\mathrm{P}$ and $\mathrm{T}$ waves. In this paper, we propose an efficient method for extraction of characteristic points of ECG signal. The method is based on a nonlinear dynamic model, previously introduced for generation of synthetic ECG signals. For estimating the parameters of model, we use an Extendend Kalman Filter (EKF). By introducing a simple AR model for each of the dynamic parameters of Gaussian functions in model and considering separate states for ECG waves, the new EKF structure was constructed. Quantitative and qualitative evaluations of the proposed method have been done on Physionet QT database (QTDB). This method is also compared with another EKF approach (EKF17). Results show that the proposed method can detect fiducial points of ECG precisely and mean and standard deviation of estimation error do not exceed two samples $(8 \mathrm{msec})$.
\end{abstract}

Index Terms - Extended Kalman Filter (EKF), Electrocardiogram (ECG), Characteristic Waves, Fiducial Point Extraction, Segmentation

\section{INTRODUCTION}

Electrocardiogram (ECG) is a non-invasive, safe and quick method for cardiac disease diagnosis. Fiducial Points (FPs) in an ECG signal are the location of peak, onset and offset of waveforms which have clinically useful information for physicians. "Fiducial Point Extraction" and "Segmentation" of ECG can be a first step in many ECG analysis tasks to determine as accurately as possible the peak, onset and offset locations of the ECG waves.

Up to now, different methods have been used for detecting the QRS complex. Some of them were discussed in [1]. These methods are based on mathematical functions, filtering

This work partially supported by scholarship of French Embassy. approaches (digital filters, adaptive filters), different mathematical transformations (Wavelet, Hilbert) and classification methods (neural network approaches, Support Vector Machine (SVM), fuzzy C-means algorithm) [1]. Some methods have also been used for $\mathrm{P}$ and $\mathrm{T}$ wave delineation and estimation such as Partially Collapsed Gibbs Sampler (PCGS) [2, 3], Hidden Markov Models (HMM) [4] and mathematical morphology methods [5].

A nonlinear dynamical model for generation of synthetic ECG signals has been recently developed by McSharry et al. [6]. Sameni et al. [7] transformed this model and proposed an Extended Kalman Filter (EKF) algorithm for denoising ECG signals ("EKF2"). Sayadi et al. modified the EKF2 framework and added parameters of ECG dynamical model as states to EKF2 and introduced the "EKF17" approach [8, 9]. They also described a Gaussian wave-based state space model whose each characteristic wave of ECG has been considered as a state ("EKF4") [10].

In this paper, we propose a method for detection the fiducial points of ECG signal. In our method by taking the idea of EKF4 and EKF17 approaches, we introduce a simple AR model for parameters of Gaussian functions in ECG dynamical model and also consider three separate states for ECG waves. In brief, we consider 25 parameters of ECG signal as states of an EKF and we will find peak, onset and offset of all characteristic waves (QRS complex, $\mathrm{P}$ and $\mathrm{T}$ waves) of ECG signal. For validation of our method, we will use QT database (QTDB) [11, 12] which has ECG signals with cardiologist annotations. We also compare our method with another EKF approach which has 17 states (EKF17).

Due to space limitations, basics of EKF are not discussed in this paper. Details of them can be found in [13, 7, 14]. In this paper we will benefit of previous EKF approaches ("EKF2", "EKF17" and "EKF4"). These approaches are discussed in Section 2. In Section 3, we explain our proposed method ("EKF25" approach) for fiducial points extraction. In section 4, we present the results of applying the proposed method for ECG signals of QT database. Finally, discussion and conclusions are provided in Section 5. 


\section{PREVIOUS EKF APPROACHES}

McSharry et al. [6] have proposed a synthetic ECG generator, which is based on a nonlinear dynamic model. Details of this model can be found in [6]. Sameni et al. [7] transformed these dynamic equations into the polar form to obtain a simpler compact set, with the simplified discrete form shown as:

$$
\left\{\begin{array}{l}
\varphi_{k+1}=\left(\varphi_{k}+\omega \delta\right) \bmod (2 \pi) \\
z_{k+1}=-\sum_{i} \delta \frac{\alpha_{i} \omega}{b_{i}^{2}} \Delta \theta_{i} \exp \left(-\frac{\Delta \theta_{i}^{2}}{2 b_{i}^{2}}\right)+z_{k}+\eta
\end{array}\right.
$$

where $\Delta \theta_{i}=\left(\varphi_{k}-\theta_{i}\right) \bmod (2 \pi), \delta$ is the sampling time, $\eta$ is a random additive noise that models the inaccuracies of the dynamic model and the summation over $i$ is taken over the number of Gaussian functions used for modeling the shape of the ECG. The $\alpha_{i}, b_{i}$ and $\theta_{i}$ terms in (1) correspond to the amplitude, angular spread and location of the Gaussian functions and $\omega$ is the angular velocity represents the $R R$ interval variability.

Sayadi et al. extended EKF2 framework and added parameters of 5 Gaussian functions in (1) as states to EKF2. In fact in this approach, 2 states were the same as in EKF2 and 15 other states were added, so that the method was called "EKF2+15" or briefly "EKF17" approach. This approach was used for ECG denoising, compression [8] and beat segmentation of normal ECG signals [9].

Sayadi et al. also described a Gaussian wave-based state space model [10] whose each characteristic wave, i.e. P, QRS and $\mathrm{T}$ has been considered as a state. As this structure had 4 states, they called it "EKF4" and used it for ECG arrhythmia detection especially PVC detection. In this model they considered two Gaussian functions for $\mathrm{P}$ and $\mathrm{T}$ waves.

\section{OUR PROPOSED METHOD}

In this paper, we modify the previous EKF approaches. Discrete state and observation equations of our proposed model are defined in (2) and (3), respectively.

$$
\left\{\begin{array}{l}
\varphi_{k+1}=\left(\varphi_{k}+\omega \delta\right) \bmod (2 \pi) \\
P_{k+1}=-\sum_{i \in\left\{P_{1}, P_{2}\right\}} \delta \frac{\alpha_{i k} \omega}{b_{i k}^{2}} \Delta \theta_{i k} \exp \left(-\frac{\Delta \theta_{i k}^{2}}{2 b_{i k}^{2}}\right)+P_{k}+\eta_{P} \\
C_{k+1}=-\sum_{i \in\{Q, R, S\}} \delta \frac{\alpha_{i k} \omega}{b_{i k}^{2}} \Delta \theta_{i k} \exp \left(-\frac{\Delta \theta_{i k}^{2}}{2 b_{i k}^{2}}\right)+C_{k}+\eta_{C} \\
T_{k+1}=-\sum_{i \in\left\{T_{1}, T_{2}\right\}} \delta \frac{\alpha_{i k} \omega}{b_{i k}^{2}} \Delta \theta_{i k} \exp \left(-\frac{\Delta \theta_{i k}^{2}}{2 b_{i k}^{2}}\right)+T_{k}+\eta_{T} \\
\alpha_{i, k+1}=\alpha_{i, k}+u_{j, k}, j=\{1, \cdots, 7\} \\
b_{i, k+1}=b_{i, k}+u_{j, k}, j=\{8, \cdots, 14\} \\
\theta_{i, k+1}=\theta_{i, k}+u_{j, k}, j=\{15, \cdots, 21\} \\
i \in\left\{P_{1}, P_{2}, Q, R, S, T_{1}, T_{2}\right\} \\
\Phi_{k}=\varphi_{k}+v_{1 k} \\
s_{k}=P_{k}+C_{k}+T_{k}+v_{2 k}
\end{array}\right.
$$

In this model, the first state is the phase of the ECG. The second, third and forth ones are the different waves of ECG which are separately considered as a state. The parameters of the Gaussian functions are considered as hidden-state variables (states 5 to 21) with first order AR dynamics but without corresponding observations. It is obvious that the ECG observation can be defined as a summation of $P, C$ and $T$ states. In fact this model is an extension of "EKF4" approach and we can call it "EKF4+21" or briefly "EKF25".

Figure 1 shows the blockdiagram of EKF25 approach for finding peak, onset and offset of normal ECG waves. At first, all states of the model are estimated by EKF25 approach. The proposed method for finding the peak of waves, consists of three steps:

- Using "peak detection" method (finding the Maxima) for estimated waves $(\hat{P}, \hat{C}$ and $\hat{T})$ and finding their peaks which are called $P_{P}, P_{C}$ and $P_{T}$. These peaks are considered as the first group candidates for final peak points of ECG.

- $\theta_{i} \mathrm{~s}$ (peaks of Gaussian functions) are 7 states which are estimated by EKF25 and are considered as the second group candidates for final peak points of ECG.

- Using a decision rule like (4) to find the final peak points of ECG $\left(\Theta_{P}, \Theta_{R}\right.$ and $\left.\Theta_{T}\right)$, which $s_{k}$ is the observed (original) ECG signal.

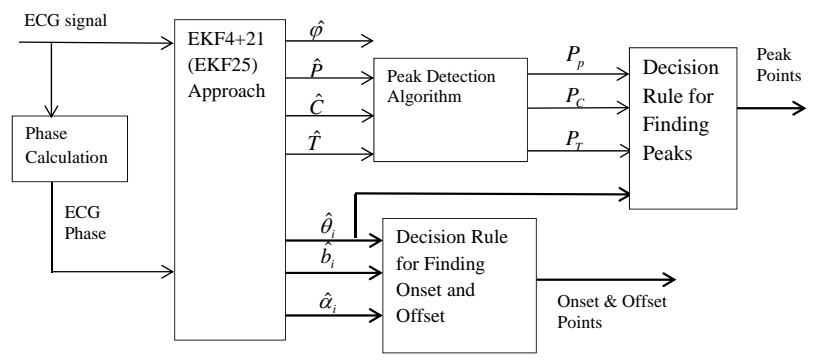

Fig. 1. Blockdiagram for the proposed EKF25 Approach.

$$
\begin{aligned}
& \Theta_{P}=\operatorname{argmax}\left(s_{k}\left(\theta_{P 1}\right), s_{k}\left(\theta_{P 2}\right), s_{k}\left(P_{P}\right)\right) \\
& \Theta_{R}=\operatorname{argmax}\left(s_{k}\left(\theta_{R}\right), s_{k}\left(P_{C}\right)\right) \\
& \Theta_{T}=\operatorname{argmax}\left(s_{k}\left(\theta_{T 1}\right), s_{k}\left(\theta_{T 2}\right), s_{k}\left(P_{T}\right)\right)
\end{aligned}
$$

In order to find the onset and offset of the waveforms, the proposed method consists of two steps:

- Finding the onset and offset of $P_{1}, P_{2}, Q R S, T_{1}$ and $T_{2}$ by using (5). In this equation same as [9], we take advantage of the spread parameter $\left(b_{i}\right)$ and use the approximately $99 \%$ confidence bound for the termination of Gaussian functions.

$$
\begin{aligned}
& P_{j_{\text {on }}}=\theta_{P_{j}}-3 b_{P_{j}}, \quad P_{j_{\text {off }}}=\theta_{P_{j}}+3 b_{P_{j}}, j \in\{1,2\} \\
& Q R S_{\text {on }}=\theta_{Q}-3 b_{Q}, Q R S_{o f f}=\theta_{S}+3 b_{S}, \\
& T_{j_{\text {on }}}=\theta_{T_{j}}-3 b_{T_{j}}, \quad T_{j_{\text {off }}}=\theta_{T_{j}}+3 b_{T_{j}}, j \in\{1,2\}
\end{aligned}
$$


- Using a decision rule like (6) to find the final onset and offset of $\mathrm{P}$ and $\mathrm{T}$ waves. $\beta_{i}, \gamma_{i}, i=\{1, \cdots, 4\}$ are real positive coefficients and by using these averaging rules, the Gaussian properties of waves can be preserved. Equations which are used for finding the onset and offset of QRS complex are same as (5) and are not changed in this step.

$$
\begin{aligned}
& P_{\text {on }}=\beta_{1} P_{1 \text { on }}+\gamma_{1} P_{2 \text { on }}, \quad P_{\text {off }}=\beta_{2} P_{1 \text { off }}+\gamma_{2} P_{2 \text { off }} \\
& T_{\text {on }}=\beta_{3} T_{1 \text { on }}+\gamma_{3} T_{2 \text { on }}, T_{\text {off }}=\beta_{4} T_{1 \text { off }}+\gamma_{4} T_{2 \text { off }}
\end{aligned}
$$

Some steps of discussed procedure are same as EKF17 approach but in fact, there are two main differences between EKF17 and EKF25. The first difference is that EKF25 approach, considers two Gaussian functions for $\mathrm{P}$ and $\mathrm{T}$ waves. The second difference is that in our approach, we define three separate states for ECG waves. On the one hand, these differences have advantage for us. For example for finding the peak of $\mathrm{P}$ wave, we make a decision between the estimated $\theta_{P 1}, \theta_{P 2}$ and $P_{P}$ and for finding the $P_{o n}$ we make a decision between the estimated $P_{1 o n}$ and $P_{2 o n}$. So we can achieve more exact results. On the other hand, in some cases making a correct decision between the estimated parameters is not simple. In general $\beta_{i}$ and $\gamma_{i}$ coefficients in (6) can be determined by mathematical calculations or by "trial and error" methods. Here, we determine these parameters by "trial and error" methods and they are not constant for all ECG signals and must be determined experimentally for each ECG signal.

\section{RESULTS}

For validation of our method, we use QT database which has ECG signals with cardiologist annotations. All records of database were sampled at $250 \mathrm{~Hz}$. Details can be found in $[11,12]$. We also compare our proposed method with previous EKF approach (EKF17). For EKF17 implementation, we use the model presented in [15].

By following the procedure of figure 1 and using equations (4)-(6), fiducial points of ECG have been detected. As we said before, values of $\beta_{i}$ and $\gamma_{i}$ used in (6) are determined experimentally for each ECG signals. These values for three normal signals are reported in tabel 1. Figures 2 and 3 show orignal labels annotated by expert (up), the results of FPs extraction by EKF25 (middle) and EKF17 (down) approaches for records "sel16539" and "sel301" respectively. Red, blue and black points show the onset, peak and offset of waves respectively. Record "sel16539" is a normal signal and in figure 2, we see that EKF25 detect FPs more precisely than EKF17. Record 'sel301' has a biphasic T wave (two consecutive negative and positive $\mathrm{T}$ waves). In this figure, estimated $\theta_{T 1}$ and $\theta_{T 2}$ are shown as two peaks of T-wave and equation (4) was not used for finding the T-wave peaks of this signal. As EKF25 approach considers two Gaussian functions for $\mathrm{T}$ wave, so in this figure we see that it can detect both peaks of $\mathrm{T}$ wave precisely whereas EKF17 can not detect them.
Table 1. Values of $\beta$ and $\gamma$ coefficients used in (6).

\begin{tabular}{|l|c|c||c|c||c|c||c|c||}
\hline record No. & $\beta_{1}$ & $\gamma_{1}$ & $\beta_{2}$ & $\gamma_{2}$ & $\beta_{3}$ & $\gamma_{3}$ & $\beta_{4}$ & $\gamma_{4}$ \\
\hline sel16795 & 0.6 & 0.4 & 0.6 & 0.4 & 0.1 & 0.9 & 0.2 & 0.8 \\
\hline sel16786 & 0.9 & 0.1 & 0.9 & 0.1 & 0.9 & 0.1 & 0.9 & 0.1 \\
\hline sel16539 & 0.6 & 0.4 & 0.2 & 0.8 & 0.8 & 0.2 & 0.2 & 0.8 \\
\hline
\end{tabular}
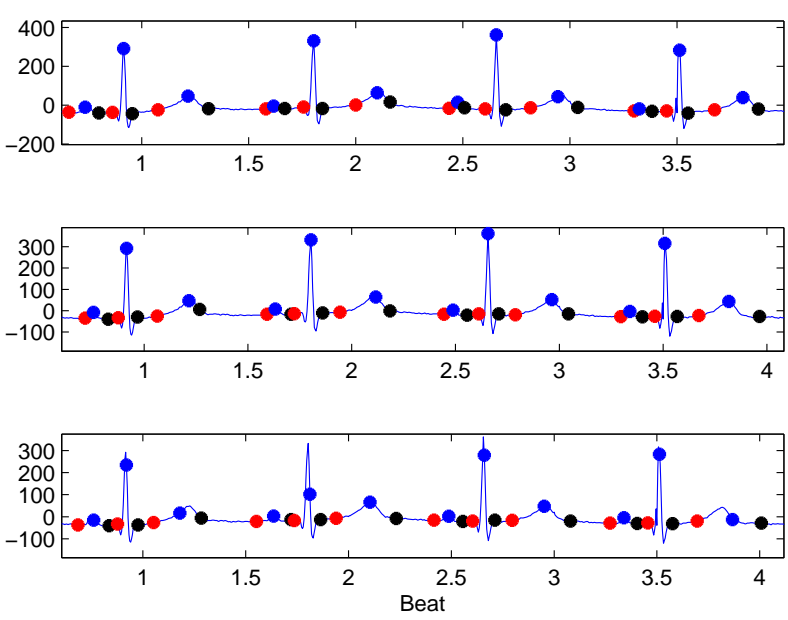

Fig. 2. (a) Original FPs (b) Estimated FPs by EKF25 (c) Estimated FPs by EKF17 for record "sel16539".

For quantitative evaluation of our proposed method, we calculate time differences between cardiologist annotations (considered as ground truth) and results of the proposed method for two normal ECG signals ("sel16795" and "sel16786"). Figure 4 shows the absolute estimation error of EKF17 and EKF25 for all the nine FPs. In this figure, beats 1 to 30 are related to "sel16795" and beats 31 to 60 are related to "sel16786". We can see that EKF25 can detect FPs with good precision and for some FPs such as $P_{\text {off }}, T_{\text {on }}, T_{\text {peak }}$ and $T_{\text {off }}$ the result of EKF25 is better than EKF17. Mean (m) and statndard deviation (SD) of estimation errors for
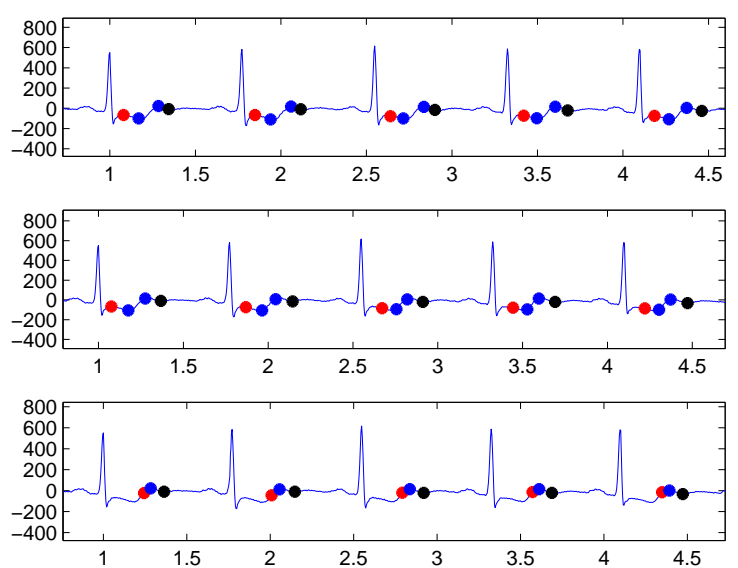

Fig. 3. T wave Parameters: (a) Original (b) Estimated by EKF25 (c) Estimated by EKF17 for record "sel301" 
these signals are given in tabel 2. We can see that for EKF25, " $m$ " and "SD" values do not exceed two samples $(8 \mathrm{msec})$ and also for some FPs such as $P_{o f f}, Q R S_{o n}, T_{o n}$ and $T_{o f f}$ error of EKF25 is very smaller than EKF17. Figure 5 shows the $T_{\text {off }}$ error estimation of "sel16539" for both EKF approaches. RR interval variations of this signal is also shown. We see that both EKF approaches are sensitive to RR interval and in beats with large RR interval variations, estimation error is high.
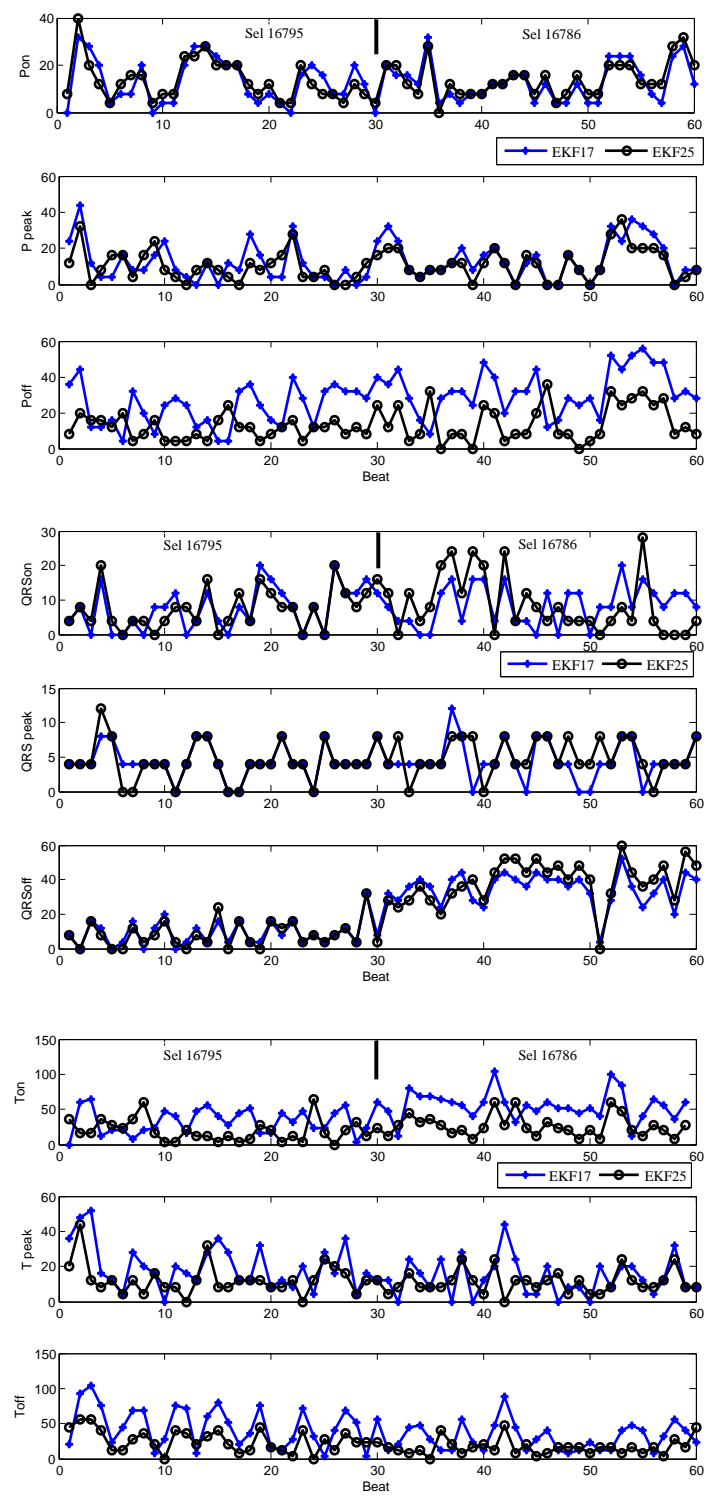

Fig. 4. Absolute error (msec) for onset, peak and offset of $P$ Wave (up), QRS Complex (middle) and T Wave (down).

\section{DISCUSSION AND CONCLUSIONS}

In this paper, we proposed a method for extracting fiducial points of ECG signal which is based on a nonlinear dynamic
Table 2. Mean and SD of errors (msec) between estimated FPs and manual annotations for two normal ECG signal.

\begin{tabular}{|l|c|c|}
\hline & \multicolumn{2}{|c|}{$m \pm S D(m s)$} \\
\hline FPs & EKF25 & EKF17 \\
\hline P on & $-0.36 \pm 3.95$ & $-0.81 \pm 3.92$ \\
\hline P peak & $2.08 \pm 2.68$ & $2.75 \pm 3.1$ \\
\hline P off & $1 \pm 3.71$ & $6.77 \pm 3.52$ \\
\hline QRS on & $-0.06 \pm 2.73$ & $0.78 \pm 2.42$ \\
\hline QRS peak & $0.15 \pm 1.06$ & $0.12 \pm 0.95$ \\
\hline QRS off & $4.44 \pm 2.94$ & $3.74 \pm 2.62$ \\
\hline T on & $0.84 \pm 5.68$ & $10.32 \pm 6.02$ \\
\hline T peak & $1.64 \pm 2.84$ & $2 \pm 4.73$ \\
\hline T off & $1.24 \pm 5.82$ & $9.04 \pm 6.72$ \\
\hline
\end{tabular}
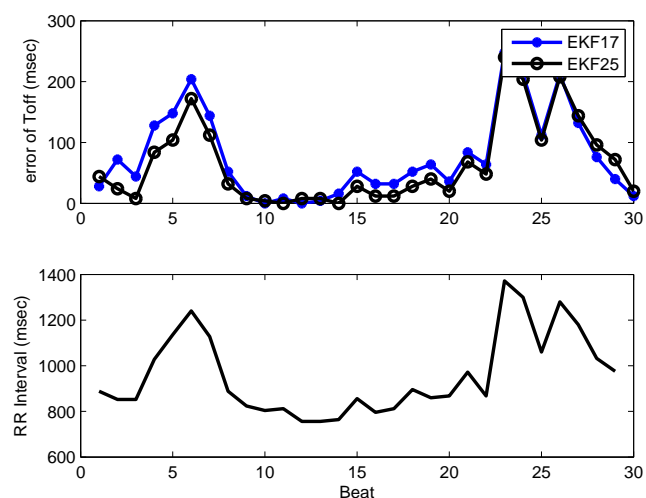

Fig. 5. RR interval variation and error of estimated Toff

model and it is an extension of EKF4 approach. By introducing a simple AR model for each of the 21 dynamic parameters of the Gaussian functions and considering separate states for ECG waves, new EKF structure was constructed.

Quantitative and qualitative results show that EKF25 approach can detect all the nine FPs (peak, onset and offset of P, QRS and T) and does not miss any one. The mean and standard deviation of estimation error of this method for all FPs do not exceed two samples $(8 \mathrm{msec})$ and in some cases, its results are better than EKF17. It can also find the T waves of a signal with bi-phasic T wave. Altought EKF25 model considers 25 states, it has a similar computational cost as EKF17.

In this model, we consider $\omega$ (angular velocity) as a process noise in EKF structure and its mean is defined by averaging RR-interval of the whole signal. By this definition, we see that for signals with major RR-interval deviations estimation error becomes high. So future work will include modifying the EKF25 approach and considering angular velocity of ECG as a new state (like [14]). In EKF25 approach, we determine $\beta_{i}$ and $\gamma_{i}$ coefficients experimentally. So another work will include finding a mathematical method for determining value of these coefficients. In addition, the proposed approach can be used for analyzing other ECG databases especially abnormal signals and signals which have asymmetrical $\mathrm{P}$ and $\mathrm{T}$ waves. 


\section{REFERENCES}

[1] B. Kohler, C. Hennig, and R. Orglmeister, "The principles of software QRS detection: Reviewing and comparing algorithms for detecting this important ECG waveform," IEEE Engineering in Medicine and Biology, pp. 42-57, February 2002.

[2] C. Lin, C. Mailhes, and J. Y. Tourneret, "P- and T-wave delineation in ECG signals using a bayesian approach and a partially collapsed gibbs sampler," IEEE Transaction on Biomedical Engineering, vol. 57, no. 12, pp. 2840-2849, December 2010.

[3] C. Lin, G. Kail, J. Y. Tourneret, C. Mailhes, and F. Hlawatsch, "P and $\mathrm{T}$ wave delineation and waveform estimation in ECG signals using a block gibbs sampler," in Proc. IEEE Int. Conf. on Acoust., Speech and Sig. Proc. (ICASSP), Prague, Czech Republic, May 2011, pp. 537-540.

[4] N. P. Hughes, Probabilistic Models for Automated ECG Interval Analysis, Ph.D. thesis, Department of Engineering Science, University of Oxford, 2006.

[5] Y. Sun, K. L. Chan, and Sh. M. Krishnan, "Characteristic wave detection in ECG signal using morphological transform," BMC Cardiovascular Disorders, September 2005.

[6] P. E. McSharry, G. D. Clifford, L. Tarassenko, and L. A. Smith, "A dynamic model for generating synthetic electrocardiogram signals," IEEE Trans. Biomed. Eng., vol. 50, no. 3, pp. 289-294, Mar. 2003.

[7] R. Sameni, M. B. Shamsollahi, C. Jutten, and G. D. Clifford, "Nonlinear bayesian filtering framework for ECG denoising," IEEE Trans. Biomed. Eng., vol. 54, no. 12, pp. 2172-2185, Dec. 2007.

[8] O. Sayadi and M. B. Shamsollahi, "ECG denoising and compression using a modified extended kalman filter structure," IEEE Trans. Biomed. Eng., vol. 55, no. 9, pp. 2240-2248, Sep. 2008.

[9] O. Sayadi and M. B. Shamsollahi, "A model-based bayesian framework for ECG beat segmentation," Physiol. Meas., vol. 30, pp. 335-352, March 2009.

[10] O. Sayadi, M. B. Shamsollahi, and G. Clifford, "Robust detection of premature ventricular contractions using a wave-based bayesian framework," IEEE Trans. Biomed. Eng., vol. 57, pp. 353-362, February 2010.

[11] $Q T$ Database [online], Available: http://www.physionet.org/physiobank/database/qtdb/.
[12] P. Laguna, R. G. Mark, A. Goldberg, and G. B. Moody, "A database for evaluation of algorithms for measurement of QT and other waveform intervals in the ECG," IEEE, Computers in Cardiology, vol. 24, pp. 673-676, 1997.

[13] S. M. Kay, Fundamentals of statistical Signal Processing:Estimation Theory, Prentice Hall PTR, 2003.

[14] M. Akhbari, M. B. Shamsollahi, and C. Jutten, "ECG denoising using angular velocity as a state and an observation in an extended kalman filter framework," in 34th Annual International Conference of the IEEE EMBS, San Diego, California, USA, Aug. 2012, pp. 2897 2900.

[15] O. Sayadi, "Model-based ECG processing (denoising, compression and classification)," M.S. thesis, Sharif University of Technology, Faculty of Electrical Engineering, August 2007. 